\title{
Rules Based Intersection Collision Avoidance System for V2X Safety
}

\author{
Than Than Yu, Tin Maung Wynn and May Zin Oo
}

Department of Computer Engineering and Information Technology

Mandalay Technological University

Mandalay, Myanmar

\begin{abstract}
The biggest problem associated with the increased use of private and public transport is the increasing number of accidents on the roads. Most of the intersection collision avoidance systems use vehicle-to-infrastructure communication to avoid accidents at urban intersections. However, they are costly because additional roadside infrastructure must be installed, and they suffer from problem-related to real-time information delivery. The proposed system namely, Rules-based Intersection Collision Avoidance System (RICA) is a novel application using GPS technology for vehicle-to-everything (V2X) safety. The distance to the intersection is calculated and a time-tointersection index is computed to establish the risk of a collision. The proposed system was achieved through simulations. It is potential as a new intersection collision avoidance system for V2X safety based on vehicular ad hoc network (VANET) communication. The result reveals that the proposed system can effectively prevent the occurrence of traffic crashes at an intersection.
\end{abstract}

Key Words: Intersection Collision Avoidance, RICA, V2X Safety, VANET, GPS.

\section{INTRODUCTION}

Nowadays, road safety has emerged as the biggest challenges in the world, especially with the high dependence of people on vehicles and the growth of traffic problems. Therefore, this situation of vehicle increment has also led to an increase of traffic congestion and accidents every year. The more increased vehicles on the road, the higher congestion and probability of accidents happened. According to the available statistical data published by WHO in 2017 concerning road accidents in Myanmar reached 10,527 or $2.6 \%$ of total deaths. The age adjusted Death Rate is 20.10 per 100,000 of population ranks Myanmar \#80 in the world [1]. There were a total of 17,451 traffic accidents nationwide in 2018, resulting in 5,184 deaths and 26,741 injuries, according to the statistical data of Myanmar Traffic Police Force published 23 January 2019 [2]. Most of the car accidents happen at intersections owing to blind areas resulting from high-rise buildings, trees and invisible conditions such as foggy or heavy rain in urban areas. These problematic blind areas at intersections can be solved by using connected safety-communication systems [3, 4].

One of the previous research methods to solve the blind area at intersections proposed the installation of sensors on the road surface to test dangerous situations and notify drivers when they exist [5]. Another research method proposed installing radar sensors on traffic lights at intersections to test other vehicles and inform drivers of dangerous conditions [6]. However, such collision avoidance systems are based on V2I communication and require installing sensors on the road surface. Moreover, they lack of the real-time capability available with V2V communication. Therefore, intersection collision avoidance systems based on VANET communication are sought. In order to solve these limitations, rules based intersection collision avoidance system is proposed using VANET communication.

In this paper, the proposed system focused on two-vehicle, three-vehicle, and four-vehicle collision avoidance scenario at an intersection to determine whether the control system is needed to protect an accident. This system prevents a collision through manually control by actuating slow down the vehicle with lower priority, but not steering, and assuming drivers follow nominal paths as predefined by the driving lanes. In this case, a control action is necessary to prevent collision, and the slowdown 
command is applied to some of the vehicles in a coordinate fashion so that some vehicles enter the intersection only after the other vehicles have existed. After the crash has been prevented, the drivers regain to control the speed of the vehicle.

Most of the vehicle collision avoidance systems are developed based on ITS concept. Previously intersection collision avoidance systems are focusing on a specific condition such as vehicle to bicycle, vehicle to vehicle and vehicle to pedestrian and not consider lane priority and direction (turn left, straight and turn right). So, the proposed RICA system will address the collision at intersection for V2X safety using VANET communication.

The rest of this research is organized as follows. Section II presents about the proposed RICA system, section III briefs about conflict point and traffic rules. Section IV discusses the simulation results and discussion and section V concludes the paper.

\section{PROPOSED RULES BASED INTERSECTION COLLISION AVOIDANCE SYSTEM}

This paper considers a case of intersection collision scenario as shown in Figure 1, in which four vehicles in different directions are approaching at an intersection and can potentially collide.

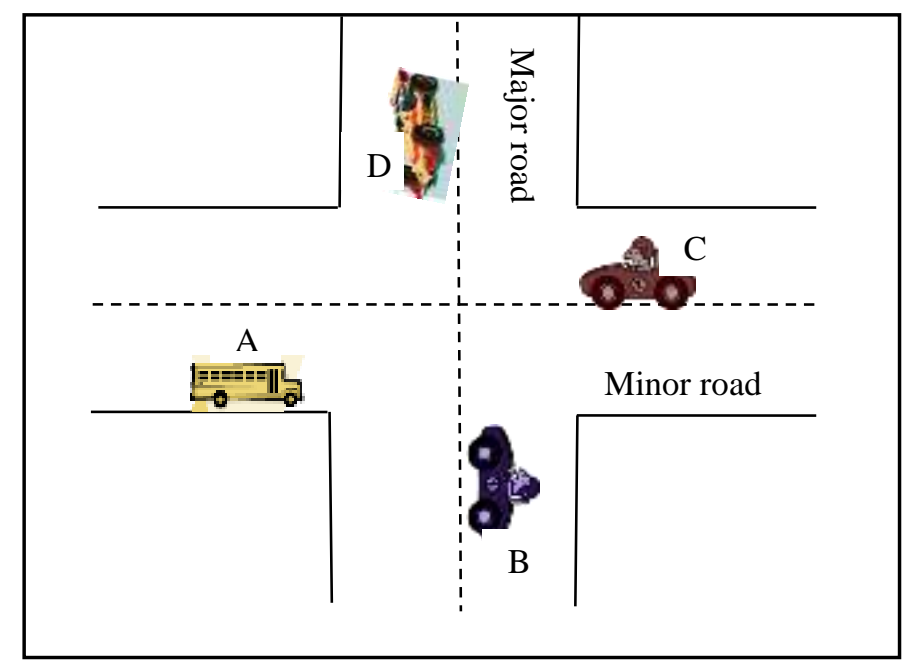

Figure 1: A case of intersection collision scenaeio

A collision may occur for a number of reasons, including a distracted driver not seeing the incoming vehicle from other directions, under estimating the vehicle speed, and violationg red light or stop signs. Collision between four vehicles are prevented by reducing the speed of some of vehicles which are on minor road, or turn left or turn right direction without controlling vehicle steering. Assuming each vehicle is installed with GPS for state information (absolute position, direction and speed), V2X communication and the ability to automatically actuate the speed control. The GPS system used in this simulation accurately establishes the position within an error range of $40 \mathrm{~m}$. The proposed system evaluates a time-to-intersection (TTI) index to establish the level of risk of collision at the intersection. TTI is the time required for a vehicle to reach the intersection. The TTI for two vehicles is calculated using as in (1) and (2). The proposed system states that the risk is high, even the TTI value is small.

$$
\begin{aligned}
& \operatorname{TTI}_{\text {host }}(\mathrm{s})=\frac{\text { Intersection distance }}{\text { host vehicle speed }} \text { host } \\
& \operatorname{TTI}_{\mathrm{n}}(\mathrm{s})=\frac{\text { Intersection distance }_{\mathrm{n}}}{\text { host vehicle speed }_{\mathrm{n}}}
\end{aligned}
$$

The distance between host vehicle and intersection, and the distance between target vehicle and intersection are calculated using as in (3) and (4) respectively. The intersection collision avoidance system is designed to evaluate the degree of risk with 
varying the $\mathrm{TTI}_{\text {host }}$ values when the absolute value of difference between the $\mathrm{TTI}_{\text {host }}$ of the host vehicle and $\mathrm{TTI}_{\mathrm{n}}$ of the target vehicle is less than a certain distance, $\mathrm{TTI}_{\text {threshold }}$, as formulated in (5). Here, " $\mathrm{n}$ " refers to the target vehicle [7].

$$
\begin{aligned}
& \text { Intersection distance }_{\text {host }}=R_{n} \cdot \cos \left(\left|R A_{n}\right|\right) \\
& \text { Intersection distance }{ }_{\mathrm{n}}=\mathrm{RD}_{\mathrm{n}} \cdot \sin \left(\left|\mathrm{RA}_{\mathrm{n}}\right|\right) \\
& \left|\mathrm{TTI}_{\text {host }}-\mathrm{TTI}_{\mathrm{n}}\right| \leq \mathrm{TTI}_{\text {threshold }}
\end{aligned}
$$

In [8], a procedure to calculate the time to collision between two vehicles is presented. Data that are considered are the initial positions of the vehicles, their speeds and directions, together with the assumption that the two vehicles are at ideal points.

$$
\mathrm{TTC}(\mathrm{s})=\frac{\text { Relative distance }}{\text { Relative speed }}
$$

In order to take into account a safety margin that compensates the simplifications made, factor $\delta$ is considered, setting the following relationship to consider collision:

$$
\left|\mathrm{TTI}_{1}-\mathrm{TTI}_{2}\right|<\delta
$$

The terms $\mathrm{TTI}_{1}$ and $\mathrm{TTI}_{2}$ are the times required for the vehicles 1 and 2 to reach the intersection point. The $\delta$ factor is the safety parameter. Therefore, for considering that a collision has occurred $\mathrm{TTI}_{1}=\mathrm{TTI}_{2}$ is not required. The higher the $\delta$ value is, the more conservative the algorithm becomes. Figure (2), (3) and (4) describe pseudo code of the proposed system, depending on the number of vehicles calculate relative distance (RD) and time-to-intersection (TTI) between adjacent vehicles.

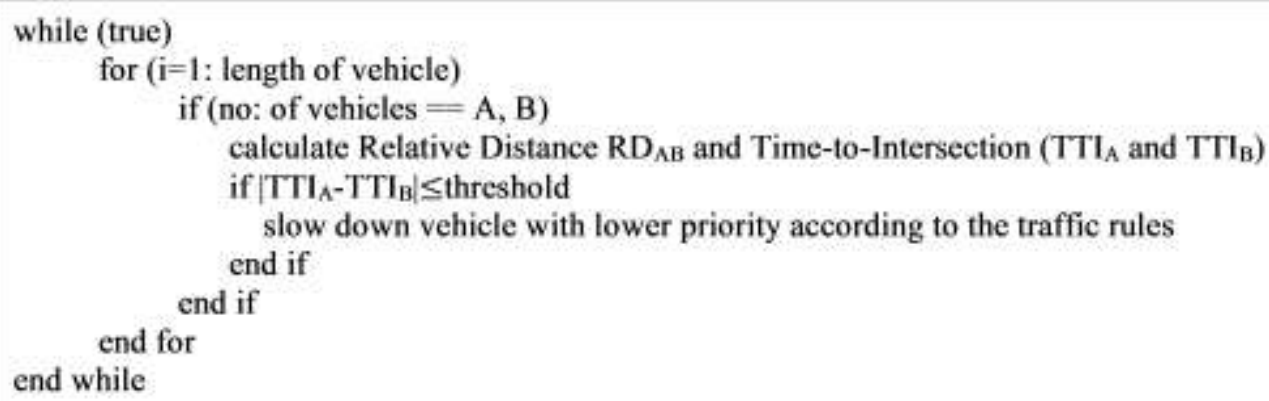

Figure 2: Pseudo code for two vehicles collision avoidance system 
while (true)

for ( $i=1$ : length of vehicle)

if (no: of vehicles ==A, B, C)

calculate Relative Distance $\mathrm{RD}_{\mathrm{AB}}, \mathrm{RD}_{\mathrm{AC}}$

if $\mathrm{RD}_{\mathrm{AC}}<\mathrm{RD}_{\mathrm{AB}}$

calculate Time-to-Intersection $\left(\mathrm{TTI}_{\mathrm{A}}\right.$ and $\left.\mathrm{TTI}_{\mathrm{C}}\right)$

if $\left|\mathrm{TTI}_{\mathrm{A}}-\mathrm{TTI}_{\mathrm{C}}\right| \leq$ threshold

slow down vehicle with lower priority according to the traffic rules end if

else if $\mathrm{RD}_{\mathrm{AB}}<\mathrm{RD}_{\mathrm{AC}}$

calculate Time-to-Intersection $\left(\mathrm{TTI}_{\mathrm{A}}\right.$ and $\left.\mathrm{TTI}_{\mathrm{B}}\right)$

if $\left|\mathrm{TTI}_{\mathrm{A}}-\mathrm{TTI}_{\mathrm{B}}\right| \leq$ threshold

slow down vehicle with lower priority according to the traffic rules end if

else $\mathrm{RD}_{\mathrm{AB}}==\mathrm{RD}_{\mathrm{AC}}$

calculate Time-to-Intersection $\left(\mathrm{TTI}_{\mathrm{A}}\right.$ and $\mathrm{TTI}_{\mathrm{B}}$ and TTIc $)$

if $\mathrm{TTI}_{\mathrm{i}} \leq$ threshold

slow down vehicle with lower priority according to the traffic rules end if

end if

end if

end for

end while

Figure 3: Pseudo code for three vehicles collision avoidance system 


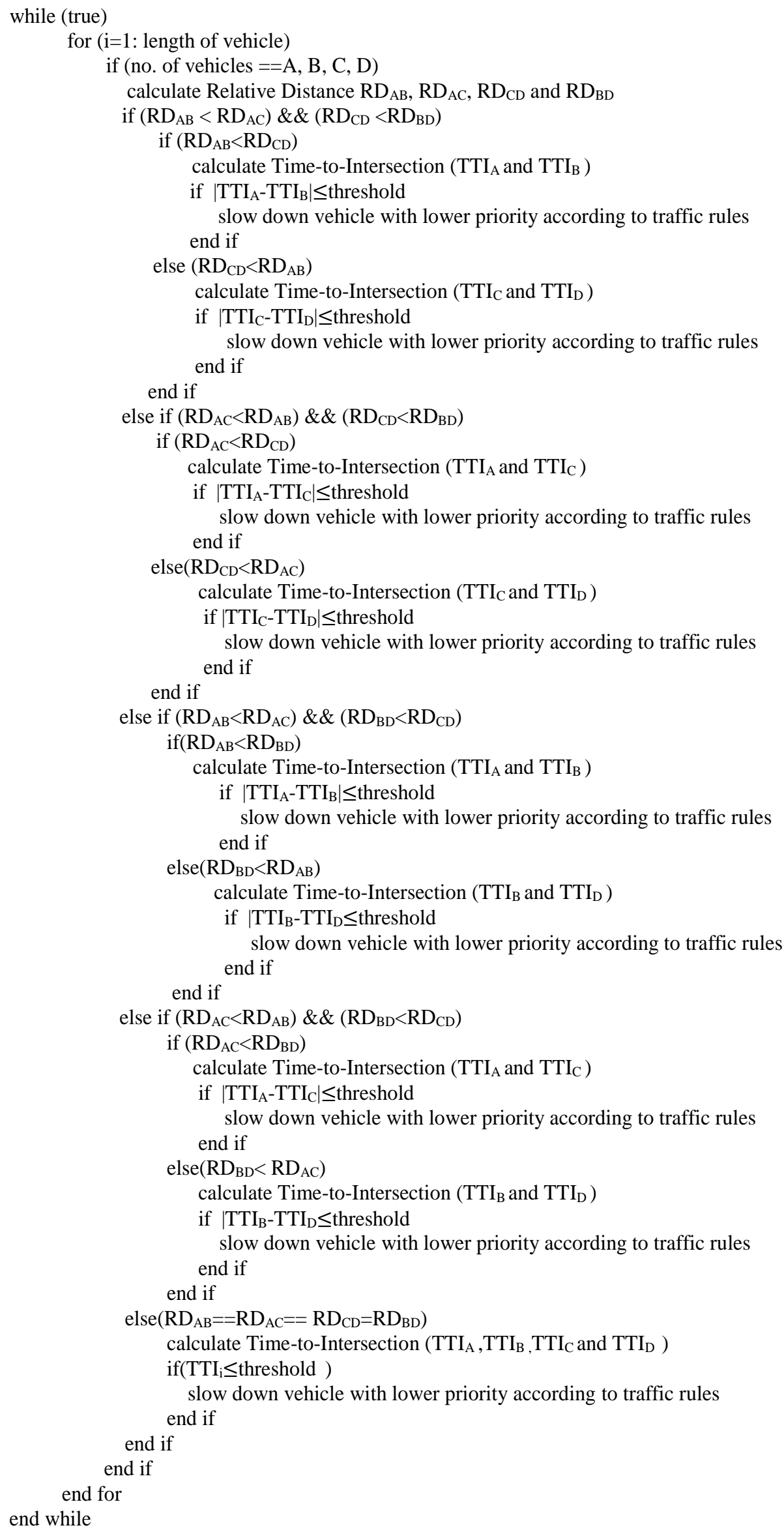

Figure 4: Pseudo code for four vehicles collision avoidance system 
The flowchart of proposed system design is also shown in Figure 5. In the proposed system design, the vehicles types are identified depending on their previous speed and if the vehicles reached in the warning zone the system generates warning alert to each vehicle.

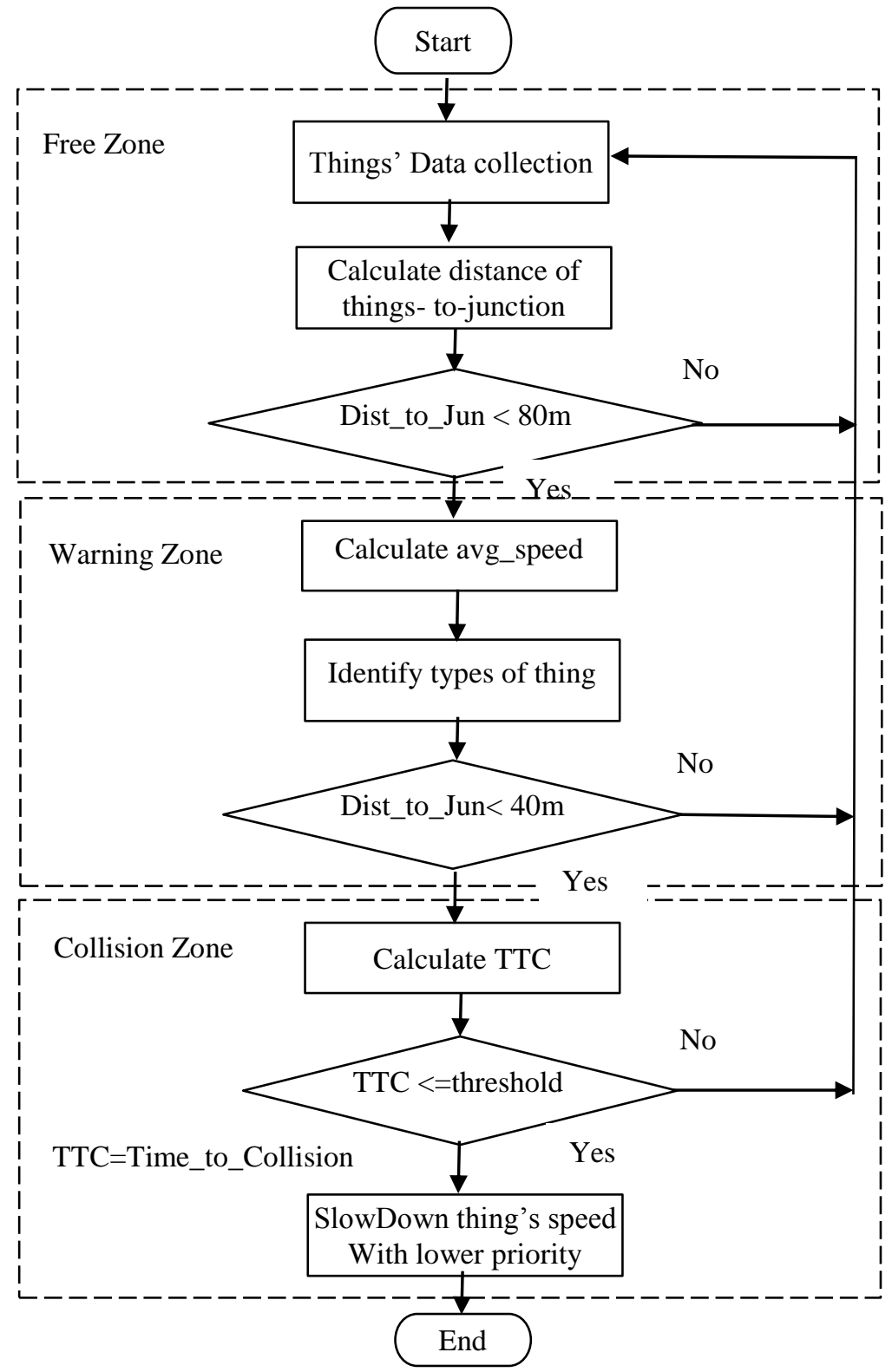

Figure 5: Flow chart of proposed system design

This system only considers three possible collision avoidance scenarios: vehicle-to-vehicle, vehicle-to-motorbike and vehicle-to-pedestrian ( V2V, V2B and V2P) at the intersection and distinguishing the types of vehicles. The purpose of every collision avoidance systems is to alert the drivers about the existence of unexpected or invisible vehicles. The system should provide a reliable real time alerting system that is capable of alerting the driver, and gives the driver time to react, as well. The proposed system that addresses the injuries posed by unexpected and invisible conditions with the ultimate aim of reducing vehicle collisions. The proposed system collects the speed, direction and position of each vehicle within the coverage area. The coverage area can be represented by either distance or time.

This system detects vehicles approaching at an intersection and captures all the required data for collision prediction. If the probability of collision is high, a warning system is activated to alert the drivers of possible collision. In this case, a control action is needed to prevent a collision, the vehicle with lower priority slows down its speed according to the traffic rules so that the vehicle with higher priority has existed from the intersection when it enters the intersection. After the crash has been prevented, 
the driver regains its speed.

\section{CONFLICT POINT SIMPLIFYING}

The vehicles from different directions converge in intersection, where the overlapping portion of the east-west and northsouth direction forms the conflict zone. The passage of vehicles at intersection is regarded as a competition where different vehicles enter conflict zones and compete for the access lines. At this point, the conflicts can be avoided by computing the relative distance between adjacent vehicles and calculating time-to-Intersection (TTI). When TTI value less than threshold value slows down vehicles with lower priority as defined by proposed traffic rules from each line. At a result, the intersection collision can be avoided. When the vehicles pass through the intersection, there will be three different routes and the driving route from different directions will conflict. Figure 6 shows the conflict point distribution of intersection for each route.

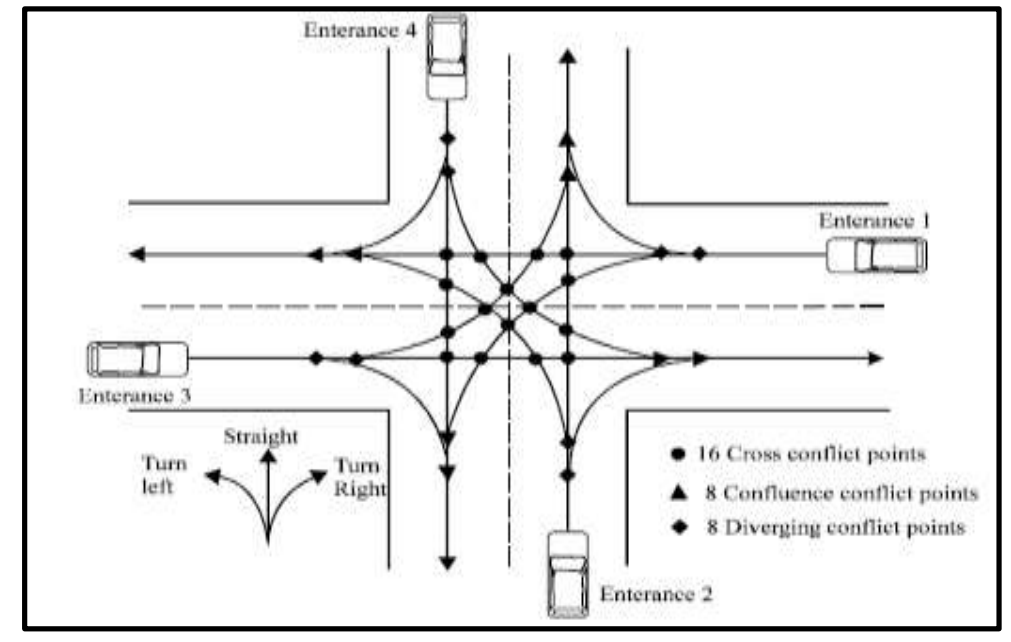

Figure 6: Distribution map of conflict point in the intersection [9]

In the intersection, there are 4 entrances from 4 directions, and vehicles from each entrance have three routes. Hence, there will be 12 different lines totally which produce 32 conflict points including 16 cross conflict points, 8 confluence conflict points and 8 diverging conflict point.

\subsection{Simplified traffic rules}

Figure 7 shows that there are many conflict points in the intersection, the conflict rules becomes more complex. The rule of first-in, first-pass will cause the frequent switching of the occupied lane, whereas the rule of conflict-free vehicle first-pass will cause the problem that the one driveway resources will always be occupied and the vehicle from the other lane cannot pass. Therefore, the intersection is divided into two parts east-west and south-north and it will simplify the intersection point and greatly reduce the probability of occurrence of the above problems. In this case, the direction from east to west is called Major Road and from south to north is called Minor Road as described in Figure 7. There are 6 conflict points for each direction, including in each cross, confluence and split and the total number of the conflict point is only 12 which is suitable for the follow-up research of the conflict resolution. 

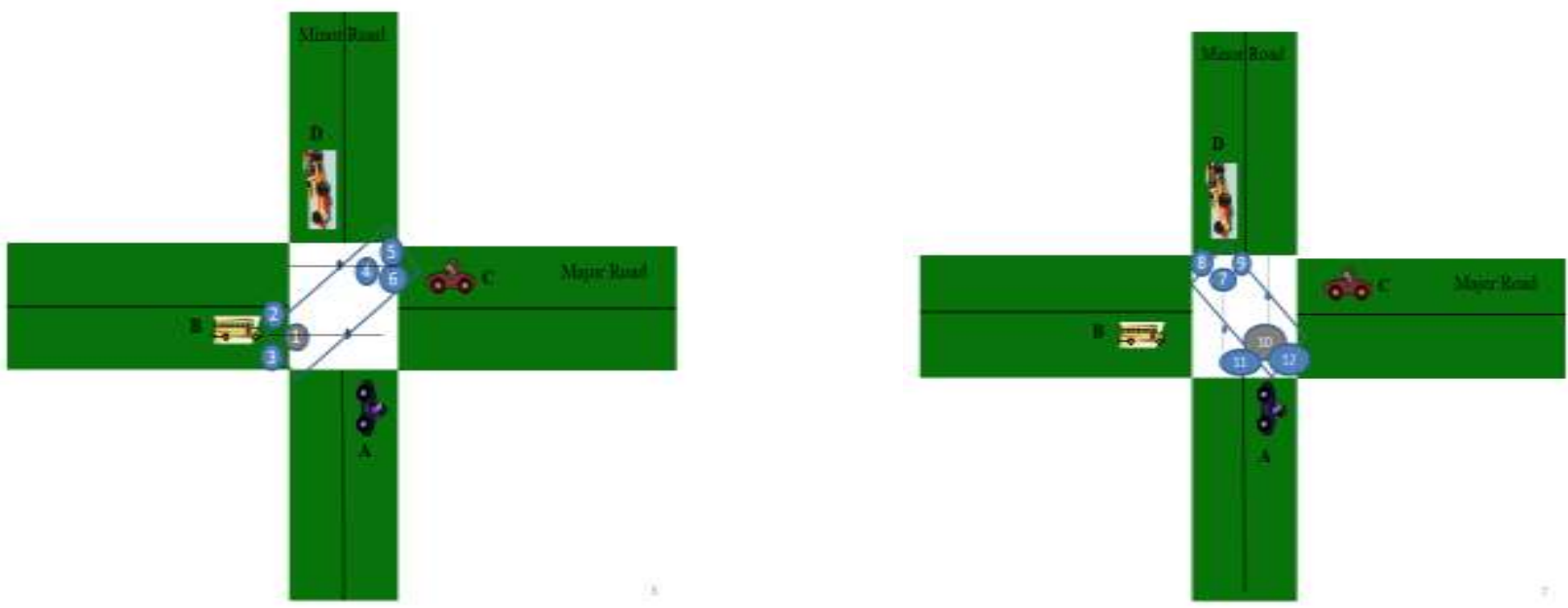

Figure 7: Conflict distributions of vehicles from east-west and south-north direction

There are three driving directions, turn left, go straight and turn right (without the situation of U-turn), for each vehicle at intersection. Thus, all possibilities of vehicles from 4 directions is indicated by the number 1-12. More specifically, vehicles that are turning left can be indicated by 2, 6, 9 and 11 whereas, turning-right vehicles can be indicated by 3, 5, 8 and 12. Finally, vehicles that are going straight can be indicated by 1, 4, 7 and 10. In this paper, major road and straight direction are set first priority and turning right is set second priority and turning left is set last priority respectively. The whole system is operated in this rules.

\section{RESULTS AND DISCUSSION}

In order to verify the proposed RICA system, an urban intersection scenario was developed using Urban Mobility simulator (SUMO). The proposed system creates a road network manually edited with NETEDIT and the junction and intersection are manually created which are saved as a net file. SUMO provides information about the road network environment like routes and vehicle properties. It is seen that SUMO is suitable for the simulation as compared to MATLAB. However, SUMO does not offer user the feature to compute and implement algorithm into it. On the other hand, MATLAB is suitable for VANET algorithm development.

TraCI4Matlab implemented as Traffic Control Interface (TraCI) application level protocol, built on top of the TCP/IP stack, so that the application developed in MATLAB, which is the client, can access and modify the simulation environment provided by the server, SUMO. TraCI4Matlab not only allows MATLAB to take control of SUMO objects such as vehicles, traffic lights, etc, but also provides users a testbed to evaluate traffic predictive control and dynamic route assignment among others. To run the simulation, SUMO's configuration file is needed which includes all the names of file such as net.xml and rou.xml.

In this system, the lanes' area is defined three zones such as free zone, warning zone and accidental zone. The vehicle within the free zone is set blue color, the vehicle within warning zone is set yellow color and the vehicles within accidental zone is set red color respectively. In Figure 8 (a) shows the simulation result of two vehicles collision avoidance scenario. When a pedestrian requests to enter the zebra crossing by pushing the button, the vehicles start to decelerate. In particular, the pedestrians move at a maximum speed of $2 \mathrm{~m} / \mathrm{s}$. Figure 8 (b) describes the simulation result of three vehicles collision avoidance scenario. In this case, the vehicle on major road is driving straight direction, the vehicle on reverse major road is driving turn right direction and the vehicle on minor road is driving turn left direction respectively. According to the proposed traffic rules the vehicle with higher priority pass through the intersection. Figure 8 (c) illustrates the simulation result of four vehicle collision avoidance scenario, where the vehicles on major road are driving with turn right and turn left directions and the vehicles on minor road are also driving with turn right and turn left direction. According to the traffic rules, vehicles with lower priority (turn left direction) slows down its speed, so that the vehicles with higher priority (turn right) direction pass through the junction. 


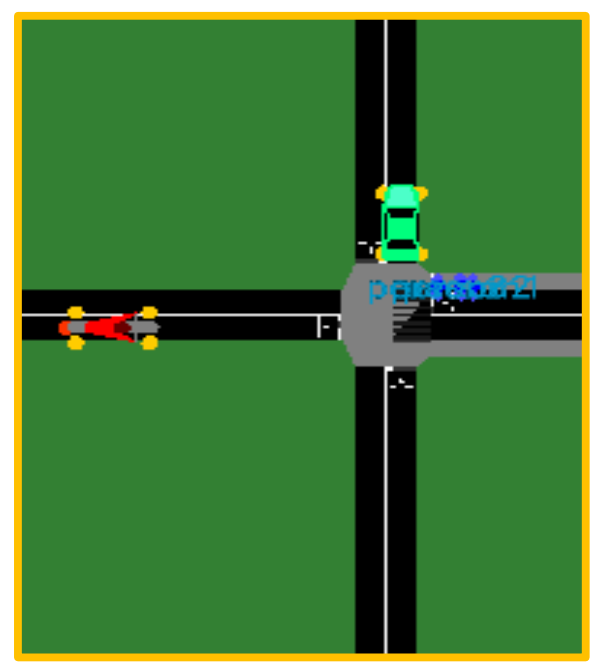

(a)

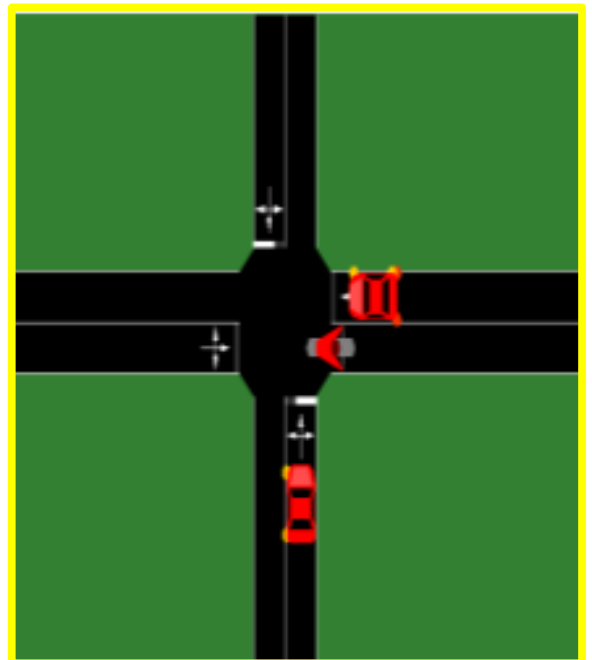

(b)

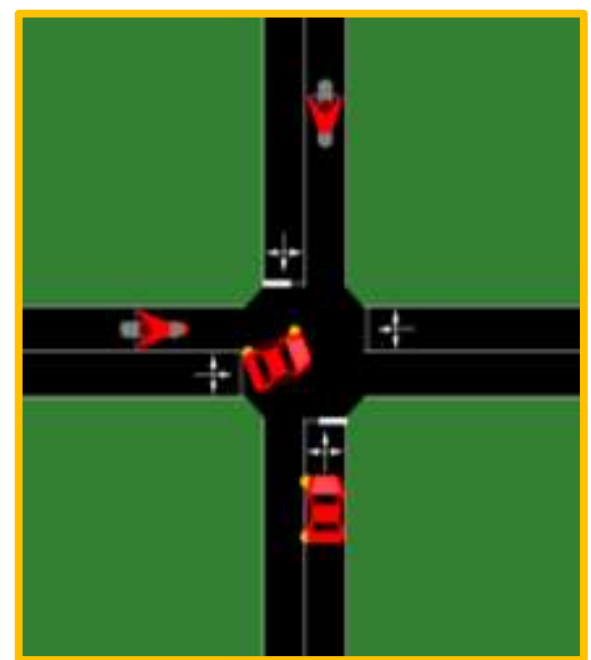

(c)

Figure 8: Collision avoidance scenario (a) two vehicles (b) three vehicles (c) four vehicles

The proposed RICA system was verified through the possibility of degree of risk by comparing the Time-to-Collision (TTC), threshold value, using traffic rules and without using traffic rules. The degree of risk was divided into three levels: 1,2 and 3. The threshold value 3 was defined as the lowest level of risk, the threshold value 2 as intermediate risk, and the threshold value 1 as the highest risk as shown in table 1 . The error rate of vehicles (A, B) are: TTC $\leq 3$ is $67.84 \%, \mathrm{TTC} \leq 2$ is $62.3 \%$ and TTC $\leq 1$ is $41.86 \%$. The error rate of vehicles $(\mathrm{A}, \mathrm{C})$ are: TTC $\leq 3$ is $75.81 \%, \mathrm{TTC} \leq 2$ is $75.3 \%$ and TTC $\leq 1$ is $17.76 \%$. The error rate of vehicles $(\mathrm{C}, \mathrm{D})$ are: TTC $\leq 3$ is $79.63 \%$, TTC $\leq 2$ is $78 \%$ and TTC $\leq 1$ is $0.86 \%$. The error rate of vehicles $(\mathrm{B}, \mathrm{D})$ are: TTC $\leq 3$ is $75.99 \%$, TTC $\leq 2$ is $75.06 \%$ and TTC $\leq 1$ is $38.47 \%$ respectively as shown in Figure 9.

Table 1. Definition of degree of risk according to changes in TTC with/without using traffic rules

\begin{tabular}{llll}
\hline Degree of Risk & low & medium & High \\
\hline TTC $[\mathrm{s}]$ & $\leq 3$ & $\leq 2$ & $\leq 1$ \\
\hline
\end{tabular}

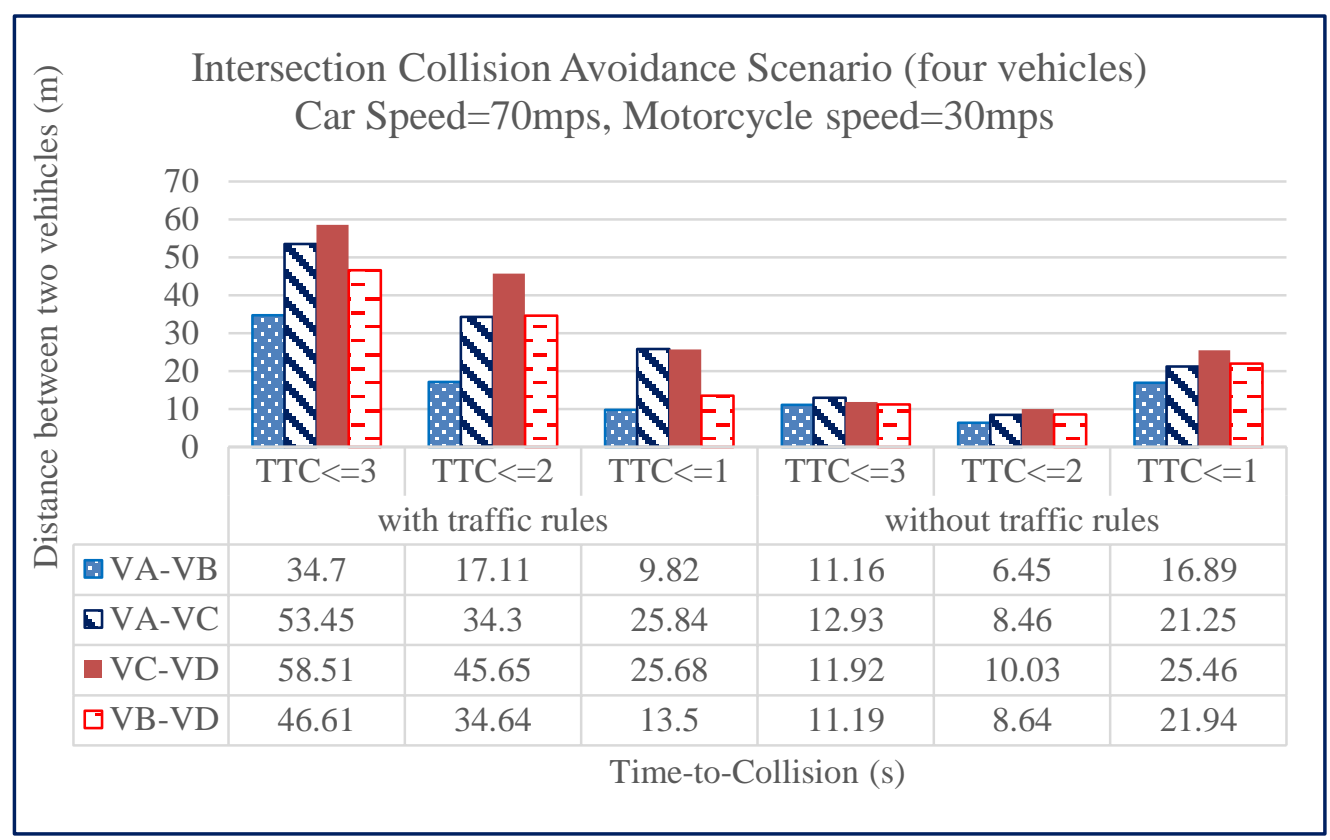

Figure 9: Simulation result with/without using (RICA) system 
International Journal of Advances in Scientific Research and Engineering (ijasre), Vol 5 (10), October-2019

\section{CONCLUSION}

In this paper, rules based intersection collision avoidance system for V2X safety is presented using VANET communication. Firstly, the system identifies the type of vehicles depending on their previous speed and put priority on lanes by identifying either major road or minor road and directions (turn left, straight, turn right),in which straight is higher priority than turning direction. RA and RD were also computed using data from target vehicle and host vehicles received from v2x communication. After computing the distance from the host and target vehicles to the intersection, the TTI was calculated to define the degree of risk. When the possibility of collision is high, the vehicle with lower priority slows down its speed according to the proposed traffic rules so that the vehicle with higher priority has passed through the junction. The lower the TTI value, the higher the possibility of collision rate. The proposed system uses mobility component, SUMO and TraCI4Matlab. The results reveal that the degree of risk of collision is significantly higher when the distance between two vehicles is nearer. This system can be extended for networking component, V2X communication using popular network simulation tool such as OMNET++ and Veins.

\section{REFERENCES}

1. "MYANMAR: ROAD TRAFFIC ACCIDENTS", available at https://www.worldlifeedpectancy.com/myanmar-roadtraffic-accidents.

2. Anonymous, https://elevenmyanmar.com/news/5184-traffic-related-deaths-26741-injuries-reported-last-year.

3. D.Caveney and W. Bunbar, "Cooperative driving: Beyound V2V as an ADAS sensor", 2012 IEEE Intelligent Vehicle Symposium. IEEE (2012), pp. 529-534.

4. T.Y. Byun, "An Performance Measurements of Inter-vehicle Communication System over Wireless WANs", International Journal of Control and Automation, 3 (2010), pp. 41-54.

5. F.Basma, Y. Tachwali and H. Refai, "Intersection collision avoidance system using infrastructure communication", 2011 $14^{\text {th }}$ International IEEE Conference on Intellignet Transporation Systems (ITSC). IEEE, (2011), pp. 422-427.

6. K. Lee, S. Jo, J. Kwon, T. Hong and K. Park, "Development of V2I-Based Intersection Collision Avoidance System", Proceedings of Conference for Korea Institute of ITS, (2013), pp. 90-96.

7. H. Cho and B. Kim, "Cooperative Intersection Collision Warning System based on Vehicle to Vehicle communication," Contemporary Engineering Sciences, Vol.7, no.22, 2014, pp. 1147-1154.

8. Jiménez, F.; Naranjo; J. E.; García, F, (2013) “An Improved Method to Calculate the Time-to-Collision of Two Vehicles”, International Journal of Intelligent Transportation Systems Research, 11(1), pp. 34-42.

9. L. Wenhui et al, (2014). "Study on Cooperative vehicles Infrastructure Collision Avoidance in Unsignalized Intersection using Simplified Conflict table”, Information Technology Journal, 13(2), pp. 231-241 